\title{
REGIONES E INFRAESTRUCTURA EN LA INTEGRACIÓN SURAMERICANA*
}

\section{Regions and infrastructure in South America integration}

\author{
Edgar Vieira Posada ${ }^{* *}$
}

Recibido: 27 de abril de 2013. Aprobado: 2 de octubre de 2013.

\section{RESUMEN}

El tema del desarrollo territorial y regional ha vuelto a adquirir importancia en el contexto de la globalización contemporánea, donde elementos conceptuales de aglomeración en entornos de competencia imperfecta y de nuevas teorizaciones como la nueva geografía económica, se unen a un trabajo intenso de desarrollo de regiones en Europa y en otras latitudes, mientras Suramérica ha quedado rezagada por el poco avance en estos temas en los procesos de integración de la Comunidad Andina y del Mercosur. Sin embargo, la reciente formalización de la Unión de Naciones Suramericanas (Unasur) y el asumir a través del Consejo Suramericano de Infraestructura y Planeamiento (Cosiplan) la agenda de proyectos prioritarios de la que estaba encargada la Iniciativa para la Integración de la Infraestructura Regional Suramericana (IIRSA), abre la posibilidad de vincular las acciones de desarrollo de las regiones con proyectos de infraestructura determinados como prioritarios para Suramérica en el 2011 a ser desarrollados al 2022, pues sin superar la falta de infraestructura será difícil incorporar las variables locales y regionales al desarrollo suramericano.

PALABRAS CLAVES: Regiones, integración, Suramérica.

Este artículo forma parte del proyecto de investigación código 40001: "El desarrollo de regiones en la Unión Europea y su aplicabilidad al nuevo ordenamiento territorial de Colombia”, de la línea de investigación sobre globalización y gestión. Grupo de Investigación en Innovación y Gestión Empresarial, Colegio de Estudios Superiores de Administración (CESA). Bogotá, Colombia.

** Colegio de Estudios Superiores de Administración -(CESA), Centro de Estudios sobre Globalización e Integración. Bogotá, Colombia. Correo electrónico: edgar.vieira@cesa.edu.co. 


\begin{abstract}
The issue of territorial and regional development has once again acquired importance in the context of contemporary globalization. Conceptual elements of agglomeration in environments of imperfect competition, and new theorizations like that of New Economic Geography, join an intense work of development of regions in Europe and elsewhere, while South America has been left behind by the little progress on these issues in the processes of integration of the Andean Community and Mercosur. However, the recent formalization of the Union of South American Nations (UNASUR) and that the South American Council of Infrastructure and Planning (COSIPLAN) has taken on the agenda of the priority projects of the South American Initiative for the Integration of Regional Infrastructure (IIRSA), which opens the possibility of linking the development of regions with certain infrastructure projects, that have been determined as priorities for South America in 2011, to be developed until 2022, because without overcoming the lack of infrastructure it would be difficult to incorporate the local and regional variables to the South American development process.
\end{abstract}

KEY WORDS: Regions, integration, South America.

\title{
I. INTRODUCCIÓN
}

La globalización contemporánea generalizada a partir de la década de 1990 reintrodujo la temática de lo territorial como variable a ser considerada para lograr una inserción mundial más competitiva, pero la respuesta mediante este instrumento, a través de los procesos de integración, solo logró avances significativos en la Unión Europea con una "política de regiones", organizada institucionalmente y respaldada en recursos mediante fondos estructurales que representan una tercera parte de los recursos financieros del proceso de integración europeo.

La integración suramericana, por el contrario, no ha otorgado la importancia suficiente al desarrollo de las regiones en las agendas de los procesos subregionales, pagando un alto costo, pues de haber existido políticas más activas, se habría logrado mayor participación de la sociedad civil desde lo local y regional, en lugar de la integración centralista de las capitales y se habrían adoptado mayores medidas para una mejor distribución de beneficios de la integración entre las regiones, reduciendo el desequilibrio en la distribución del ingreso que caracteriza a América Latina frente al mundo. 
Es de esperar que las nuevas funciones en infraestructura asumidas por la Unión de Naciones Suramericanas (Unasur), que antes pertenecían a la Iniciativa para la Integración de la Infraestructura Regional Suramericana (IIRSA), permitan un desarrollo en infraestructura que sirva igualmente para adelantar programas de integración de las regiones.

Hasta ahora, no son muchos los resultados de la Comunidad Andina (CAN) o del Mercado Común del Sur (Mercosur), por lo que se plantea en este escrito la necesidad de reforzar acciones para acompañar a Unasur en programas integrales de desarrollo de integración física, que contribuyan al desarrollo de políticas de las regiones en ámbitos nacionales, transfronterizos y transnacionales, pues se parte como hipótesis de que la superación de los atrasos en infraestructura en Suramérica se debe realizar teniendo en cuenta su impacto en los programas de desarrollo de las regiones e involucrando a la sociedad civil.

El artículo comienza con algunos desarrollos conceptuales en el tema de lo territorial y regional en un contexto de globalización, tiene como referente principal la experiencia europea, analiza algunos instrumentos vinculados a lo territorial en los procesos subregionales y regional suramericanos, y la proyección de nuevas acciones decididas recientemente en proyectos de la Iniciativa para la Integración de la Infraestructura Regional Suramericana con el Consejo Suramericano de Infraestructura y Planeamiento (COSIPLAN) de Unasur.

\section{ALGUNOS DESARROLLOS CONCEPTUALES EN EL TEMA DE LAS REGIONES}

Los economistas de las escuelas clásica y neoclásica no incorporaron factores relacionados con la espacialidad territorial, dejando de lado aspectos de localización productiva en el desarrollo territorial de unas regiones y no de otras, pues las concepciones de la economía clásica desarrollaban un modelo de competencia perfecta sin referencia a lo geográfico y territorial.

En la década de 1950, unos de los primeros análisis sobre los efectos de creación o desviación en el comercio en fases de integración de uniones aduaneras (Viner 1950), acompañaron aspectos teóricos clásicos y neoclásicos de creación de comercio mediante un razonamiento de competencia pura y perfecta, pero sin explicaciones adecuadas sobre 
el desarrollo de los primeros procesos de integración en Europa, donde la especialización productiva intrasectorial contradecía el intercambio intersectorial de los clásicos.

El surgimiento de los países en desarrollo a la vida independiente, al producirse el proceso de descolonización a mediados del siglo XX, dio origen en ese momento a teorías de desarrollo económico y de subdesarrollo que consideraron elementos territoriales, como la "causalidad circular acumulativa" (Myrdal 1959), teorías que fueron retomadas más tarde por la corriente de la nueva geografía económica en un solo marco teórico, con lógicas de causación circular con aglomeración de actividades que brindan ventajas de localización a una región y donde se introdujo la naturaleza intrasectorial de los intercambios y se propuso una formulación modernizada del dilema costo de distancia - economías de escala (Siröen 2004).

Esto representó reacercamientos a teorías de la década de 1950 de polos de crecimiento (Perroux 1955), de políticas económicas propias de países en desarrollo (Hirschmann 1958), de causalidad circular acumulativa (Myrdal 1959) y de etapas del crecimiento económico (Rostow 1963).

Aquellas teorías del subdesarrollo y el desarrollo económico fueron complementadas por los teóricos de la Comisión Económica para América Latina (Cepal), encabezado por su secretario Raúl Prebisch (1951) y secundado por valiosos colaboradores como Celso Furtado, quienes consideraron la necesidad de industrializar la región para romper la relación desfavorable existente en los términos de intercambio, con la incorporación de economías de escala en espacios ampliados de esquemas de integración.

La aparición en la década de 1970 de una nueva teoría del comercio internacional fue el resultado de evidencias empíricas de entornos sin ventajas comparativas, ni competencia perfecta, ni intercambio intersectorial, que dieron lugar a la consideración de economías imperfectamente competitivas, con intercambios intrasectoriales en una competencia de precios de monopolio y de sectores oligopolísticos (Siroen 2004), y con la incorporación muy significativa para el tema de las regiones de los efectos de la geografía sobre la localización de las actividades económicas.

El comercio internacional para Paul Krugman era expresión particular de la geografía económica y de configuraciones centro - periferia, características de los intercambios comerciales, los cuales podían ser explicados por los mecanismos circulares de aglomeración (Krugman 1992: 14), con corrientes de comercio intraindustrial, en procesos de integración económica competitivos en escenarios de espacios económicos ampliados. 
Las "economías de aglomeración" resultantes producen enmarcamientos en amont y en aval de las empresas ${ }^{1}$, reforzando la aglomeración de actividades en encadenamientos en estructuras espaciales concretas, y en la vigencia de factores como localización y costos de transporte. Gracias a los efectos de aglomeración surgidos con la globalización contemporánea, las actividades productivas tienden a fijarse lo más cerca posible de los lugares de consumo en función de los costos de transporte de los lugares de producción y de las zonas de población densa con poder de compra elevado, lo que a la vez conduce al problema de marginamiento de las periferias.

El proceso llega a un punto en que las fuerzas centrípetas que conducen a la aglomeración comienzan a ser compensadas por fuerzas centrífugas como los costos de la tierra, el transporte y las deseconomías externas por congestión y polución (Moncayo 2002: 12). Aparece igualmente el papel de la integración para reducir las disparidades y concentraciones que ocasionan los fenómenos de aglomeración, pues al producirse la apertura comercial se reducen los costos de comercialización con la desconcentración desde un solo centro hacia diferentes centros sectoriales especializados del mercado ampliado (Berretoni y Lucángeli 2012: 35).

El territorio deja de ser entonces un simple lugar receptor de actividades productivas para convertirse, según Pierre Veltz, en el actor, la estructura activa del desarrollo. Lo que se produjo estos últimos años puede ser llamado "reinvención del territorio", donde los gobiernos regionales y sus empresas pueden adaptarse mejor a las nuevas condiciones de un mercado ampliado (Veltz 1996). Son "las regiones que ganan" (Benko y Lipietz 1992).

Como modalidad específica de desarrollo aparece el concepto de "desarrollo económico local”, expresión de agrupamiento espacial que reúne denominaciones como economías de aglomeración, distritos industriales, entornos de innovación tecnológica, clusters, tecnopolos. Algunos de estos conceptos venían de otras épocas, como la noción de "distrito industrial" de Alfred Marshall, de la década de 1920, distrito donde se ubican empresas que trabajan un producto homogéneo con compenetración e interrelación en lo económico y social.

Estos aportes al desarrollo económico local los precisa Francisco Alburquerque como la reelaboración de la noción de distrito industrial de Alfred Marshall (1920) realizado por Becattini (1979); la noción de medio innovador definida por los equipos de

1 Términos provenientes del francés referentes a fases o actividades anteriores y posteriores, generadas en un entorno propio en el proceso productivo. 
investigación que integran el Grupo de Investigación Europeo sobre Medios Innovadores (1990); la conceptualización de la estrategia de especialización flexible desarrollada por Piore y Sabel (1984); y la discusión sobre los complejos productivos (clusters) realizada por Porter (1991) (Alburquerque 2001: 23).

En los "distritos industriales" de conocidas aplicaciones en el norte de Italia, la "acumulación o especialización flexible" de la década de 1980 de Michael Piore y Charles Sabel como contribución a la producción posfordista que impulsó la globalización, fue realizada por la pequeña y mediana empresa gracias a la utilización de máquinas flexibles y de obreros calificados que permitió producciones de productos más adaptados al consumidor en un contexto local de procesos de desarrollo autónomo.

En este papel de innovación tecnológica, el énfasis se desplazó hacia sectores terciarios avanzados, en concentraciones espaciales de carácter acumulativo (a lo Myrdal y Kaldor) en determinados espacios, en una serie de interrelaciones entre empresas y unidades de investigación, con efectos expansivos en la producción de bienes y servicios avanzados (Moncayo 2002: 16).

A los distritos industriales del norte de Italia (Toscana, Lombardía, Venecia y Emilia Romagna), especializados en ciertos sectores tradicionales de manufacturas como la industria del cuero, se agregaron distritos industriales en otras regiones europeas (Valencia en España, Flandes en Bélgica o Baden-Wurtemberg en Alemania); en los Estados Unidos (Silicon Valley, condado de Orange en California); y en América Latina distritos industriales en Nuevo León, México, y en Santa Catarina y Campiñas en el Brasil (Moncayo 2002).

Para atender necesidades en competitividad y afrontar la globalización, en la década de 1990 se desarrolló la conformación de polos de desarrollo con la denominación de clusters (Porter 1991), concentraciones geográficas de empresas e instituciones conectadas en un sector, que permiten la formación de tecnopolos como resultado de acciones gubernamentales para motivar la localización en un lugar de actividades productivas, tecnológicas y de investigación. Ello dio origen a concentraciones de "ciudades región", "ciudades tecnológicas" o "ciudades del conocimiento" (Tsukuba, Japón) y parques científicos (Cambridge), con mejores condiciones para lograr inserciones mundiales competitivas.

Estos nuevos espacios urbanos, regionales y nacionales se mezclan, según Bob Jessop, “con escalas de internacionalización, triadización, formación de bloques regionales, cons- 
trucción de redes de ciudades globales, formación de regiones transfronterizas, localización internacional, glocalización, glurbanización y transnacionalización” (Jessop 2004: 28).

Se observa cómo se han acomodado nuevos términos que recogen la mezcla de tríada con globalización (triadización), de globalización con localización (glocalización) y globalización con urbanización (glurbanización). La valorización de lo local ha dado lugar a terminología extraña como glocal (Robertson) y fragmegración (Rosenau), para expresar la pertenencia simultánea a ámbitos globales y locales, de fragmentación e integración. Como anota Edgard Moncayo: "La globalización de lo local y la localización de lo global" (Moncayo 2002: 9).

Otra contribución a la revalorización de lo regional fue la incorporación de variables de la geografía natural y ambiental a través de nociones de desarrollo sostenible y de "biorregión", con el desarrollo de la teoría del "biorregionalismo" sobre gestión de recursos de los ecosistemas, recursos naturales que han adquirido nueva relevancia en la apertura económica. La "biorregión" fue definida por el Instituto de Recursos Mundiales (WRI), la Unión Internacional para la Conservación de la Naturaleza (IUCN) y el Programa de las Naciones Unidas para el Medio Ambiente (PNUMA), como un territorio de agua y de sol en el que los límites son definidos por los límites geográficos de comunidades humanas y sistemas ecológicos.

Tal zona debe ser suficientemente larga para mantener la integridad de las comunidades biológicas, hábitats y ecosistemas de la región; sostener procesos ecológicos esenciales, tales como los ciclos nutritivos y desperdicios, migración y flujos; satisfacer las exigencias del territorio para especies claves; e incluir las comunidades humanas en el manejo, utilización y comprensión de los recursos biológicos (Guimaraes 2001: 23).

Esta incorporación del desarrollo sostenible y el biorregionalismo es producto de la universalización de la problemática medioambiental aportada por la globalización y tiene especial significado para Sudamérica, una de las regiones más ricas del planeta en biodiversidad. Alrededor de la protección y beneficio de esta riqueza podrían estructurarse proyectos de desarrollo regional en niveles subnacionales, transfronterizos y transnacionales.

\section{CONTEXTO DE GLOBALIZACIÓN}

Se ha podido apreciar la influencia del contexto de globalización en los cambios conceptuales y revalorización de lo territorial y regional, por lo cual es conveniente co- 
mentar algunos elementos del proceso globalizador. En la tercera ola de globalización contemporánea generalizada a partir de la década de $1990^{2}$, los temas de políticas territoriales y regionales volvieron a ser asuntos prioritarios para las políticas de desarrollo, a las que se les sumaron las políticas de integración con las que se procedió a dar respuesta para buscar una inserción más competitiva.

Esta exigencia de competitividad aportada por el contexto de globalización se produjo con el abandono del modelo proteccionista, en el que particularmente América Latina basó su desarrollo durante más de cinco décadas, el remplazo en muchos casos del modelo fordista de producción por producciones flexibles basadas en la innovación, la productividad y la competitividad; el desarrollo de tecnologías de punta y la necesidad de grandes inversiones en investigación, así como la revolución en los campos de los transportes y de las comunicaciones informáticas (Vieira 2012).

Todo ello complementado con la apertura generalizada de los mercados mundiales con la aparición de nuevos clientes y competidores, principalmente compañías transnacionales, gracias al impulso de tratados de libre comercio que abrieron la competencia no solo a las mercancías, sino a los servicios, acompañados de reglas de juego para la propiedad intelectual y para la inversión extranjera; la aparición de nuevos actores, en particular organismos intergubernamentales internacionales, y la organización de localidades regionales y municipales que permitirían revalorizar lo territorial y regional con el desarrollo de políticas de regiones.

El problema ya comentado es que no todas las regiones están en condiciones de seguir el ritmo y desafíos de la globalización. En este sentido se puede hacer referencia a la globalización como a una interrelación entre economías prósperas de algunas regiones de un grupo de países determinados, en contraste con regiones periféricas atrasadas. Como respuesta al centralismo político, la globalización sería un resurgimiento de las regiones subnacionales y el crecimiento de las regiones transfronterizas y transnacionales.

2 Un primer período u ola de globalización es reconocido por autores como Aldo Ferrer, Fernando Braudel, Angus Maddison, Pierre de Senarclens, Osvaldo Sunkel, Hugo Fazio en el siglo XV de despertar renacentista con grandes descubrimientos y unas relaciones comerciales intercontinentales en el auge del mercantilismo. Un segundo período ocurre en el siglo XIX, entre 1850 y 1913 (Guillermo de la Dehesa, Kevin O’Rourke, Jeffrey Williamson, David Held, Anthony McGrew), y es de gran expansión de los imperios europeos y de aparición de mejores condiciones de transporte, en una fase de claros avances del capitalismo mundial. La tercera ola o período globalizador se generaliza en la década de los noventa del siglo XX con los mandatos incorporados en el Consenso de Washington y la prevalencia del modelo neoliberal de reglas del mercado. 
En el actual contexto internacional, no son más los Estados los que hacen la competencia, son las regiones y las ciudades-región. Es más difícil para los Estados obtener su inserción en la medida en que la flexibilización de la producción y la descentralización, características del actual modelo productivo, son más fácilmente desarrollables por unidades reducidas como las regiones. Se trata de una competencia por los mercados, por la tecnología y por los capitales, y ciertamente las regiones están mejor colocadas para hacerlo de una manera competitiva y más eficaz, que la capacidad de los Estados centralizados para adaptarse a las estructuras flexibles (Vieira 2008: 220 y 217).

Ahora bien, son muy variadas las interpretaciones de la globalización, pues en la misma academia ha sido imposible lograr una interpretación que "haya alcanzado la condición de ortodoxia en los círculos académicos. Al contrario, hay teorías contrapuestas que pugnan por la supremacía" (Held y McGrew 2003: 14)3. Esto se debe a que la globalización no es un proceso lineal, sino que se dan tendencias de intensificación o de desaceleración del proceso (Fazio 2002: 42). Y es que los resultados de la globalización son complejos e incluso caóticos al ser procesos de escalas múltiples, plazos temporales y centros que funcionan en contextos estructurales precisos (Vieira 2012).

Otra característica de la globalización es su instantaneidad por la compresión del tiempo y de la distancia, con lo cual los sistemas, mercados y fenómenos culturales tienen incidencia local inmediata, gracias a lazos de interdependencia creados en un contexto mundial (Brünner 1998: 134); pues en la globalización hay una intensificación de relaciones sociales planetarias debido a una aproximación tal entre lugares distantes, que sucesos locales son influenciados por sucesos ocurridos a gran distancia y viceversa (Giddens 1994: 70).

Se produce el desdibujamiento de las fronteras o proceso de desfronterización (debordering), delante de la conformación de espacios económicos ampliados integrados más allá de las fronteras, estructurando espacios territoriales no solamente subnacionales, sino transfronterizos y transnacionales de dos o varios países. Esto realza la importancia adquirida por las regiones como medio de compensar la menor presencia del Estado-nación y de desarrollar mejores posibilidades de inserción competitiva internacional.

3 El profesor y consultor del Departamento de Estado de los Estados Unidos Manfred Steger considera que "los eruditos no solo opinan de diferentes maneras en cuanto a definiciones propias de globalización; ellos también discrepan sobre su escala, causación, cronología, impacto, trayectorias y políticas de resultados" (Steger 2009: 11). El término globalización de origen anglófono es compartido con el de autores que se refieren a este proceso como de mundialización, con la adopción de posiciones a favor o en contra, donde se destacan propuestas de otro mundo posible o altermundialización (Wievorka, Wolton). 
Todo ello indica cómo la globalización se caracteriza por mayores interdependencias y conexiones globales, donde cada vez más partes del mundo van siendo incorporadas.

El historiador Fernand Braudel, en su análisis sobre el Mediterráneo en el siglo XVI (Braudel 2005), logró demostrar la importancia que adquiere el escenario geográfico para el análisis de los desarrollos de las regiones y en su pensamiento. De acuerdo con el análisis del académico Eric Helleiner, la globalización es un fenómeno espacial donde la connotación de distancia se reduce con reconfiguraciones espaciales que desafían la territorialidad de los Estados-nación, en bloques macrorregionales y mercados ampliados con normas supranacionales, en procesos de contracción espacial en los que se involucran comunidades locales (Helleiner 2000), tal como ha sucedido con el desarrollo de políticas de regiones en espacios de integración como la Unión Europea y en menor escala el Mercosur.

La consideración de lo regional se enmarca en la existencia de niveles diferentes para ejercer la gobernabilidad, pues la globalización redujo el protagonismo de los Estados incorporando nuevos actores: en un nivel superior, las organizaciones internacionales; en un nivel intermedio, los Estados; y en un nivel más bajo, las regiones y municipios. El repliegue del Estado de la escena mundial condujo a la formación de estructuras territoriales por encima de las fronteras a nivel de macrorregiones o regiones transnacionales, regiones de diversos Estados articulados directamente con la economía internacional o con regiones de Estados vecinos como regiones transfronterizas; y por debajo de los Estados-nación: microrregiones, ciudades-región, tecnopolos o vastas áreas metropolitanas que hacen su propia inserción internacional (Vieira 2008: 238).

Gary Marks considera que en el caso de la integración europea, desde el Acta Única en la segunda mitad de la década de 1980 se produjo la emergencia de la "gobernanza multinivel" (multilevel governance) como sistema de negociación entre gobiernos en varios niveles territoriales: supranacional, nacional, regional y local, lo cual ha impulsado al nivel supranacional funciones previamente centralizadas del Estado y hacia abajo funciones al nivel local y regional, que le permite a la sociedad civil incorporarse de manera más activa al funcionamiento de la integración y el desarrollo (Marks 1993: 392). Por eso para Michael Keating se produce la existencia de "un orden político complejo en el cual la política europea se regionaliza, la política regional se europeíza y la política nacional se europeíza al mismo tiempo que se regionaliza" (Keating 1996: 68).

La jerarquización espacial de nivel global, plurinacional (bloques multinacionales), nacional y regional, permite que las regiones se vuelvan centros influyentes de re- 
gulación económica y de autoridad política, y asimismo se conviertan en el marco para nuevos tipos de comunidades sociales y nuevas aproximaciones de asuntos de ciudadanía (Scott 2001: 23), tal como lo ha demostrado la experiencia de la política de regiones de la Unión Europea o del sudeste asiático.

\section{DEFINICIONES DE REGIÓN Y POLÍTICA DE REGIONES EN LA UNIÓN EUROPEA}

Pero, ¿qué se considera región? La existencia de una región implica un territorio o territorios donde hay cierta homogeneidad en cuanto a circunstancias sociales, naturales, económicas, culturales e históricas (González 1995: 16). También puede ser un área geográfica con un mínimo desarrollo metropolitano, asociada a un área (hinterland) que funciona como marco espacial para la vida cotidiana de un grupo de gentes, en donde se genera una mezcla de actividades socioeconómicas sujetas a fuerzas de polarización (Scott 2001: 14).

La región puede ser considerada vector de modernización a nivel local al ser institución intermedia en un conjunto de relaciones verticales (centro y periferia) u horizontales (procedimientos contractuales, financiamientos cruzados), que afectan las relaciones de jerarquía entre lo transnacional y lo infranacional (Mabileau 1998: 54).

A nivel formal, la región fue definida en el artículo 1 de la Declaración sobre el regionalismo en Europa adoptada por casi 300 regiones de la Asamblea de las Regiones de Europa (ARE), el 4 de diciembre de 1996, en Basilea, Suiza, así:

La región es la entidad pública territorial de nivel inmediatamente inferior al Estado, dotada de autogobierno político. La región encuentra su reconocimiento en la Constitución o en la ley, la cual garantiza su autonomía, identidad, competencias y forma de organización [y para tal efecto] será dotada de una Constitución propia, de un estatuto de autonomía o de otras leyes que formarán parte del ordenamiento jurídico del Estado al más alto nivel (Asamblea de las Regiones de Europa 1996).

De una manera más general, según el geógrafo Olivier Dollfus puede haber distintos niveles regionales, donde el término regional comprende conceptos como la división de un territorio nacional de una cierta extensión; la región de los diplomáticos, con el reagrupamiento de países vecinos que ofrecen unidad histórica, cultural y la región for- 
mada por parcelas de territorios nacionales contiguos cuya economía y aun la vida social se restructura transgrediendo las fronteras nacionales (Dollfus 1993).

El término nivel regional comprende subconjuntos de naturaleza institucional de procesos de integración como mercados comunes, uniones económicas, zonas de libre comercio; redes de empresas que juegan con las diferencias de potencial existentes de parte y otra de una frontera (el arco Seúl - Singapur), y una variante de iniciativas de ciertas ciudades de establecer hermandades más allá de las fronteras (el triángulo Singapur-Johor en Malasia, Riau en Indonesia) (Dollfus 1993: 81-4), como lo iniciado por los países del Cono Sur en Mercociudades.

Lo importante para desarrollar formas territoriales y regionales autónomas, sean estas regiones subnacionales o transfronterizas, tecnopolos o ciudades-región, es tener sentido de un interés territorial común, lo cual lleva a interrogarse por qué ha sido tan difícil concretar la integración sudamericana donde hay tantos intereses comunes heredados de la colonización española.

En el caso de la integración europea, desde el propio preámbulo del Tratado de Roma se consideró el tema regional como la necesidad de asegurar un desarrollo armonioso al reducir diferencias entre las diversas regiones; y más recientemente en el Tratado de Ámsterdam de la década de 1990, se estableció que la Comunidad Europea se propondrá reducir las diferencias entre los niveles de desarrollo de las diversas regiones y el retraso de las menos favorecidas (Tratados de Roma y de Ámsterdam).

Al reactivarse el proceso con el Acta Única de 1986, se dio inicio a una política de "cohesión social" orientada a regiones desfavorecidas (Artículo 23 del Acta Única Europea), al hacer referencia a la noción de cohesión económica y social con el fin de "reducir las diferencias entre las distintas regiones y el retraso de las regiones menos favorecidas", en procura de un desarrollo más equilibrado. Y a partir de 1988 se formalizaron cuatro fondos estructurales: el Fondo Europeo de Desarrollo Económico Regional (FEDER), el Fondo Social Europeo (FSE), el Fondo Europeo de Orientación y Garantía Agrícola (FEOGA) y el Instrumento Financiero de Orientación de la Pesca (IFOP).

Los fondos estructurales buscan satisfacer necesidades de las regiones en las categorías de regiones desfavorecidas, zonas con desventajas específicas, grupos sociales vulnerables, colectividades locales y regionales, y países candidatos al ingreso a la Unión (Communautés Europeennes 1998). 
En 1992, el Tratado de Maastricht no solo implicó una profundización de la integración al fijar los mecanismos y procedimientos para lograr una unión económica y monetaria, sino que representó un avance en el tema de las regiones, al dotarlas de un marco institucional con la creación del Comité de las Regiones en el artículo 198 del Tratado, otorgándole reconocimiento a los gobiernos regionales y locales en el marco institucional de la naciente Unión Europea.

A partir del Tratado de Maastricht, las ayudas se incrementaron significativamente, con un aumento del 30 por ciento del presupuesto para los fondos estructurales regionales, lo que sumado a la creación del Comité de las Regiones y a la aplicación del principio de subsidiariedad, facilitó el acercamiento de las regiones a las instituciones de Bruselas.

De esta forma, el presupuesto de los fondos estructurales pasó de 8.000 millones de euros por año en 1989, a 32.000 millones de euros por año en el período 2000-2006 y a casi 50.000 millones de euros por año en el período 2007-2013. En cifras totales para estos períodos, la Unión Europea destinó a políticas de desarrollo de las regiones 213.000 millones de euros en el período 2000-2006, lo cual permitió una transferencia de más de 35 por ciento del presupuesto de la Unión Europea hacia las zonas más vulnerables; y la suma de 347.000 millones de euros, que representan más de la tercera parte del presupuesto total de la Unión Europea, para el período 2007-2013.

El trabajo europeo para el desarrollo de las regiones estuvo destinado principalmente al apoyo del ajuste estructural de las regiones atrasadas y en el período 2007-2013, al objetivo Convergencia, que tiene el propósito de estimular el crecimiento y el empleo en las regiones menos desarrolladas, con énfasis en innovación y sociedad del conocimiento, adaptación a cambios económicos y sociales, calidad y sostenibilidad medioambiental y eficiencia administrativa mediante mejores prácticas de gobernanza y de capacidades institucionales (Comunidades Europeas 2007).

Uno de los elementos destacables es el inicio de acciones en espacios transnacionales que implican pedazos de territorios nacionales que por encima de las fronteras construyen proyectos en común, dando respuesta a problemáticas no vinculadas a la territorialidad del Estado-nación. Comenzaron denominándose "Iniciativas comunitarias" en el período 2000-2006 y pasaron a ser objetivos de Cooperación Territorial Europea a partir del período 2007-2013, mediante la promoción de soluciones comunes entre diferentes territorios en materia de desarrollo urbano, rural y costero, comercio transfronterizo, desarrollo de relaciones económicas, puesta en red de pymes, medioambiente, 
infraestructura en sectores de salud, educación, cultura y turismo; redes transeuropeas; prevención de riesgos, eficiencia energética y gestión integrada del agua (Comunidades Europeas 2007).

Para esta cooperación transnacional todas las regiones son en principio elegibles, pero en el período 2007-2013 fueron seleccionadas por la Comisión en concertación con los Estados miembros, 10 zonas de cooperación transnacional localizadas en el continente europeo:

1) Periferia Norte; 2) Mar Báltico; 3) Noroeste de Europa; 4) Mar del Norte; 5) Sudoeste de Europa; 6) Costa Atlántica; 7) Espacio Alpino; 8) Europa Central y Oriental; 9) Mediterráneo; y 10) Sudeste de Europa. De esta manera se trabaja en proyectos transfronterizos al tiempo con nuevas áreas sin relación con las fronteras de los Estados, al considerar que tienen la posibilidad de desarrollar sinergias entre espacios transnacionales que decidan trabajar en común.

Como lo comenta Maria Alice Lahorgue:

El proceso de integración de la Unión Europea demostró que con el derribe de las fronteras se hizo viable una fuerte articulación entre regiones de diferentes países. Muchas veces, estas regiones no son limítrofes, pareciendo que la condición necesaria y suficiente es la existencia de intereses comunes y de una identidad económica y cultural (Lahorgue 1997: 6).

\section{INTERACCIÓN REGIONAL EN OTRAS REGIONES}

Las posibilidades de desarrollo mediante interacción entre regiones son igualmente válidas en otras regiones del mundo. Una primera tendencia es la de consolidar cierta autonomía regional al interior de los Estados, como por ejemplo en los casos de los Estados canadiense y español con las regiones autónomas de Québec o Cataluña, en el que su grado de desarrollo y el interés de resaltar diferencias frente al Estado al que pertenecen, les motiva a ser partidarias de la integración con otros países, ya que puede ser un recurso para desarrollar su autonomía (Keating 1996). Cataluña, por ejemplo, utiliza el lema "Catalunya, un país d'Europa", para significar la proyección internacional a la que aspira directamente como región autónoma. 
Estas tendencias se reflejan en el interés de constituirse en naciones regionales, naciones integrantes de un Estado, como fue planteado por grupos de Cataluña o del País Vasco en la reivindicación precisada en 1998 en la Declaración de Barcelona, de transformar a España en un "Estado plurinacional de tipo confederal". Esto lleva a un sentimiento de pertenencia en diferentes niveles territoriales, ser español por vivir en un Estado plurinacional español, ser catalán como expresión de identidad de nación y ser europeo como expresión de pertenencia a una colectividad más amplia y extendida (Loyer y Villanova 1999: 27).

Un globalófilo como Kenichi Ohmae señalaba desde la década de 1990 unidades geográficas con sentido regional en lugares tan diversos como el norte de Italia (Lombardía y Piamonte), Baden-Würtemberg o alto Rhin en Alemania, la región de Rhône-Alpes en Francia, centrada en Lyon con lazos mercantiles con Italia; la región de Languedoc-Roussillon, con vínculos con Cataluña; San Diego/Tijuana en la frontera mexicano-estadounidense, Silicon Valley/Bahía de San Francisco, Pusan y el extremo sur de la península de Corea, Hong Kong/China del Sur (Shenzhen y Guangzhou); Tokio y tres prefecturas adyacentes cuyo Producto Nacional Bruto (PNB) si fuesen un Estado los colocaría detrás de Estados Unidos y Alemania; la región japonesa de Kansai (Osaka, Kobe y Kioto, que ocuparía el sexto lugar, luego de Reino Unido); ciudades de Fukuoka y Kitakyushu en el norte de la isla japonesa de Kyushu; el triángulo del crecimiento de Singapur; Johore, Estado más meridional de Malasia y las islas de Riau; la isla malaya de Penang (gemela de Singapur) en el estrecho de Malaca que conecta con Medan, ciudad indonesia de Sumatra y Phuket en Tailandia, conformando el que se conoce como un gran triángulo del crecimiento (Ohmae 1997).

En esta tendencia mundial de insertarse al mundo desde las regiones, el problema es que aparte la frontera de México con los Estados Unidos y la ciudad de Sao Paulo, América Latina aparece excluida de estas consideraciones de potenciales desarrollos. Y acá entra el tema de infraestructura, pues el atraso en la integración física sudamericana es una limitante al trabajo que se pueda haber adelantado de desarrollo de regiones. Por eso, cualquier análisis del contexto sudamericano, debe partir de las condiciones existentes para el desarrollo de regiones y de los proyectos en materia de infraestructura.

\section{EL DESARROLLO DE LAS REGIONES EN LA INTEGRACIÓN SUDAMERICANA}

En la década de 1960 aparecieron en Sudamérica preocupaciones sobre la importancia de la regionalización para pasar del subdesarrollo al desarrollo, donde la falta de 
integración interna de los países era considerada una barrera para hacer el recorrido realizado por las economías industrializadas. Había necesidad de adelantar integración física, económica y sociopolítica, remplazando la vieja división político-administrativa correspondiente a una organización territorial propia del pasado colonial, por una nueva organización territorial en regiones (Boisier 1994).

Pero las carencias en términos de cohesión territorial, debido a la subsistencia del concepto de frontera como barrera y no como área de desarrollo comunitario, a la insuficiencia económica de regiones periféricas desprovistas de grandes ciudades que sirvan de polos de desarrollo, a la falta de estrategias de desarrollo regional, de posibilidades de gobernanza a diferentes niveles y de condiciones de acceso a redes de transportes, explicarían el atraso de Sudamérica en el desarrollo de regiones. Y en el tratamiento del tema por los procesos subregionales de integración, los avances han sido muy limitados.

\section{a. Comunidad Andina (CAN)}

En el caso de la Comunidad Andina se abordó el tema de desarrollo de las regiones apenas en el siglo XXI, luego de décadas de existencia del proceso. Fue en el 2004, en la Cumbre de San Francisco de Quito, cuando la Secretaría General planteó la participación de los espacios subnacionales en el proceso de integración andino, que condujo a una Estrategia Andina de Desarrollo y Competitividad Territorial (EADT) y en 2005 a un primer estudio sobre "Elementos para una estrategia de desarrollo territorial".

En cuanto a espacios transfronterizos, apenas cerrando el siglo XX fue que la Comunidad Andina adoptó instrumentos de desarrollo de Zonas de Integración Fronteriza (ZIF), reglamentadas mediante las decisiones 459 y 501. En la Decisión 459, de 1999, sobre "Política comunitaria para la integración y desarrollo fronterizo", se establecían como objetivos centrales:

impulsar el desarrollo económico de las Zonas de Integración Fronteriza (ZIF), considerando las particularidades de cada ZIF en las estrategias que aseguren la generación de empleo, mejoren los ingresos y eleven el nivel de vida, mediante la promoción de actividades productivas viables y el estímulo a las micro, pequeñas y medianas empresas (Consejo Andino de Ministros de Relaciones Exteriores 1999).

En la frontera colombo-venezolana, por ejemplo, como lo señalan los investigadores que realizaron el proyecto de ZIF Táchira-Norte de Santander, con la creación de esta 
zona "se aspira a la normalización y apoyo legal de la serie de relaciones translimítrofes que la población de esta frontera expone haber mantenido y desarrollado cotidianamente como resultado de la vecindad y contactos permanentes" (Bustamante y Sierra 2004), donde las numerosas interacciones entre las poblaciones de los dos lados de la frontera permitieron escoger un territorio de $3.200 \mathrm{~km}^{2}$ y una población de 1.243 .000 habitantes. Pero el distanciamiento político entre los gobiernos de Colombia y Venezuela, y la concepción distinta sobre modelo de desarrollo, ha conducido al aplazamiento de un proyecto que sería base efectiva de integración y desarrollo territorial en un espacio conjunto transfronterizo.

Una Zona de Integración Fronteriza (ZIF) es la localización precisa para que se produzcan encadenamientos de las empresas, con el resultado de aglomeraciones de actividades que se autorrefuerzan progresivamente (Bustamante y Sierra 2004: 178). El problema es que las ZIF se han quedado mayoritariamente en el papel y apenas se han adelantado unas pocas acciones aisladas, en lugar del desarrollo integral e interdependiente de los proyectos previstos para lograr la cohesión en las zonas de frontera.

La integración transfronteriza se trató de complementar con los Centros Binacionales de Atención en Frontera (CEBAF) mediante la Decisión 502, luego del fracaso relativo de los Centros Nacionales de Atención en Frontera (CENAF) y se puede considerar inaudito que después de tantos años de esfuerzos por integrarse, no se trabaje mancomunadamente en las diferentes fronteras andinas, cuando las reglas de trabajo en común en frontera son condición necesaria para el buen funcionamiento e instrumentación de los programas de apertura comercial y de integración política y social.

De otra parte, y dado el proceso de desarrollo urbano característico de los últimos tiempos, en 2003 se creó la Red Andina de Ciudades (RAC), para poner a trabajar interactivamente a 36 comunidades citadinas mediante mejor administración, mejoramiento de condiciones de vida y construcción de sociedades locales competitivas en el mundo globalizado, según la Declaración de Quito (Comunidad Andina 2006). Pero fuera del reparto inicial de unas funciones entre ciudades capitales, la Red no ha tenido continuidad en sus trabajos.

A nivel institucional, en 2004, se creó el Consejo Consultivo Andino de Autoridades Municipales (CCAAM) mediante la Decisión 585 de la Comisión Andina, para facilitar la participación de las autoridades municipales en el Sistema Andino de Integración (SAI). Solo se estableció formalmente en 2005 y tampoco presenta hasta la fecha realizaciones dignas de destacar, pues se han quedado en la instancia de la reunión buro- 
crática, pero no han pasado a la acción, concretando los objetivos acordados inicialmente (Comunidad Andina 2004).

El proceso andino por consiguiente no solo llegó tarde a los temas relacionados con las regiones, sino que no ha logrado implementar programas sólidos y continuos, lo que es un lastre para el avance del proceso en la conformación de espacios ampliados con vasos comunicantes, perdiendo la oportunidad de contar con una mayor participación ciudadana y de la sociedad civil fronteriza y con medios para mejorar la distribución del ingreso de tal manera que el ciudadano común reciba los beneficios de la integración.

\section{b. Mercado Común del Sur (Mercosur)}

En el caso del Mercosur, en 2005, se trató de seguir el ejemplo de la Unión Europea de apoyo a las regiones con la creación del Fondo para la Convergencia Estructural del Mercosur (FOCEM), en el que se contempla el desarrollo de cuatro tipos de programas: convergencia estructural; desarrollo de la competitividad; cohesión social y refuerzo de la estructura institucional y del proceso de integración, según Decisión No 18/05 (Mercosur 2005) y la organización del Programa de Integración Productiva (Decisión CMC No $12 / 08)$.

El limitante es que como este proceso no tiene instituciones supranacionales, los aportes y manejo le corresponde a los Estados miembros y no a un presupuesto comunitario en el que intervienen las regiones. Tiene a su favor el esfuerzo de financiación por los Estados más ricos, donde de acuerdo al promedio del PIB, Brasil aporta el 70 por ciento del presupuesto, pero no se puede beneficiar de aportes superiores al 10 por ciento para los proyectos de los tres primeros programas; Argentina aporta el 27 por ciento del presupuesto y no recibe más del 10 por ciento; y para los dos países más pequeños, en tamaño Uruguay y en desarrollo Paraguay, la tendencia es inversa: aportan mucho menos y reciben mucho más. Así, los aportes de Uruguay representan el 2 por ciento mientras que recibe 32 por ciento, y Paraguay hace una contribución del 1 por ciento del presupuesto del FOCEM, pero recibe casi la mitad de los recursos: el 48 por ciento (Mercosur 2005).

Hasta diciembre de 2011, el primer programa de convergencia estructural alcanzó 90 por ciento del total de proyectos aprobados y se ocupó de las regiones menos desarrolladas y de la mejora de los sistemas de integración fronteriza, mediante proyectos de 
energía eléctrica e hídrica, así como la construcción, modernización y recuperación de vías de transporte multimodal que sirven a la integración física.

El programa de desarrollo de la competitividad, 5,4 por ciento del monto de proyectos aprobado en 2011, contribuye a los procesos productivos, los mecanismos de reconversión productiva y profesional, los proyectos de integración de cadenas productivas, de investigación y desarrollo de nuevos productos y aquellos que apuntan a mejorar la calidad de la producción.

En cuanto al programa de cohesión social, 5,7 por ciento del total aprobado en 2011 implica proyectos de desarrollo social en materia de salud, enseñanza, capacitación y orientación profesionales, creación de empleo en regiones de menor desarrollo relativo y promoción del acceso a la vivienda, salud, alimentación y educación de sectores vulnerables de las regiones más pobres y de las regiones fronterizas.

En términos prácticos, un dato interesante es que en el período 2008-2011, el 94 por ciento de los fondos del FOCEM se concentraron en problemas de infraestructura física y beneficiaron regiones de menor desarrollo, como la ruta en el departamento de Concepción (Paraguay) y el proyecto de energía eléctrica en la provincia de Corrientes (Argentina), o la línea férrea en Uruguay (Berretoni y Lucángeli 2012: 33-40).

En otros frentes, ya en 1995 se había creado la Red de Ciudades de MercosurMercociudades, con una agenda de cooperación entre gobiernos locales en temas como descentralización y desarrollo sustentable, promoción de la eficiencia y transparencia de los gobiernos locales y participación de los municipios en el proceso de integración regional, creación de un ámbito institucional para las ciudades y desarrollo del intercambio y la cooperación horizontal entre las municipalidades de la región.

Y en 2004 se creó el Foro Consultivo de Municipios, Estados Federados, Provincias y Departamentos del Mercosur que comenzó sus trabajos en 2007, para estimular el diálogo y la cooperación entre las autoridades y poner en relación los representantes regionales con las directivas del proceso de integración a través de un Comité de Municipios y un Comité de Estados Federados, Provincias y Departamentos, los que a través del Grupo del Mercado Común presentarían propuestas de mejoramiento de la calidad de vida de los habitantes (Consejo Mercado Común 2004). Este Foro al menos presenta 11 reuniones de trabajo hasta 2011. 
De esta manera, el proceso de integración del Mercosur, con mucho menos años de existencia que el de la Comunidad Andina, muestra una mayor dinámica y progreso en el inicio de acciones de atención a las regiones, las cuales son fundamento indispensable para expandir el alcance y beneficios de la integración a la sociedad civil involucrada.

\section{LOS PROGRAMAS DE INFRAESTRUCTURA PARA EL DESARROLLO DE LAS REGIONES}

En el desarrollo de la infraestructura, las diferencias en los dos continentes son abismales. Mientras en la Unión Europea la política de regiones ha contribuido significativamente a que regiones de menor desarrollo se integren físicamente al continente mediante proyectos de infraestructura para unir desde el Atlántico hasta los Urales, en América Latina, a pesar de las enormes distancias que generaron núcleos poblacionales aislados, se tardó mucho tiempo en otorgarle importancia y ha contribuido al freno de los procesos de integración, pues de qué puede servir la libre circulación de mercancías y de servicios si no se dispone de condiciones adecuadas de infraestructura. Las limitadas condiciones en materia de infraestructura de medios de transporte y de comunicaciones son una de las mayores preocupaciones respecto del acompañamiento que debe recibir una política de desarrollo de regiones en América del Sur que solo posee ejes de comunicación vertical bordeando sus costas, pero sin comunicación entre el Atlántico y el Pacífico.

La solución a esta desconexión se inició en el año 2000 con la creación por los presidentes suramericanos en Brasilia de la Iniciativa para la Integración de la Infraestructura Regional Suramericana (IIRSA), en cuyo programa de trabajo quedaron incorporados cuatro ejes que tienen que ver con los territorios andinos y cinco ejes con el resto de Suramérica (IIRSA 2011). Lo que se debe analizar con mayor atención es hacia dónde van estas acciones, enmarcadas recientemente dentro de las responsabilidades de la Unión de Naciones Suramericanas (Unasur), porque no se deben evaluar solamente desde el punto de vista de infraestructura, sino incluir el impacto en el desarrollo de las regiones donde se implementen.

El Eje Andino busca utilizar la infraestructura vial, resolviendo problemas de pasos de frontera y fomentando el desarrollo en materia energética y de telecomunicaciones; el Eje del Amazonas, mejorar la navegabilidad de la cuenca amazónica, uniendo los dos océanos en un sistema multimodal entre puertos del Atlántico brasileño con los del Pacífico colombiano, ecuatoriano y peruano; el Eje Interoceánico Central debe conectar 
cinco países -Bolivia, Brasil, Chile, Paraguay y Perú- a través de medios ferroviarios y carreteros; y el Eje Perú-Brasil-Bolivia debe establecer un eje transversal a través de los territorios de estos países.

El eje de Capricornio debe articular el norte de Argentina y de Chile, el Estado de Río Grande do Sul de Brasil y la zona suroccidental de Paraguay; el Eje Guyanés, conectar Guyana, Surinam, oriente de Venezuela y el corredor Manaos-Boa Vista, en Brasil; el Eje de la Hidrovía Paraguay-Paraná, una integración fluvial entre Argentina, Bolivia, Brasil, Paraguay y Uruguay; el Eje Mercosur-Chile, unir los dos océanos por uno de los lugares de mayor movimiento comercial de América del Sur; el Eje del Sur, conectar los dos océanos a través del sur de Argentina con los puertos de Bahía Blanca y San Antonio y Chile con los puertos de Talcahuano y Puerto Montt; y el Eje Andino del Sur, conectar Bolivia con Chile y la parte oeste de Argentina hasta Tierra del Fuego (IIRSA 2011).

\section{Unión de Naciones Suramericanas (Unasur) y Consejo Suramericano de Infraestructura y Planeamiento (Cosiplan).}

La creación de la Unión de Naciones Suramericanas (Unasur) se decidió en 2006, sustituyendo a la Comunidad Suramericana de Naciones, creada en 2004, pero solo entró en funciones en 2008, procediendo a estructurar diferentes consejos por temas, uno de los cuales formalizado en 2009, es el Consejo Suramericano de Infraestructura y Planeamiento (Cosiplan).

En el Programa de Proyectos Piloto de IIRSA, la interconexión de los proyectos de infraestructura y de las regiones correspondientes requiere el desarrollo indispensable de los "pasos de frontera", tema hasta ahora trabajado insuficientemente en los procesos de integración especialmente andino, y que deberán tomar nuevo aire con las prioridades señaladas para los Proyectos Piloto de Cúcuta (Colombia) - San Antonio (Venezuela), Desaguadero (Perú - Bolivia), Foz de Iguazú (Brasil) - Ciudad del Este (Paraguay), Cristo Redentor (Argentina y Chile), y Puerto Unzué (Argentina) y Fray Bentos (Uruguay).

La formalización de Cosiplan, que remplazó al Comité de Dirección Ejecutiva de IIRSA, le ha permitido reformular los ejes de infraestructura de los primeros 10 años, en un concepto de ejes de integración y desarrollo (EID), cuyos criterios fueron aprobados en reunión de abril de 2011 en Río de Janeiro. 
En junio de 2011, en reunión en Bogotá, se reunieron los grupos técnicos ejecutivos de los nueve ejes de integración y desarrollo para revisar los proyectos según los criterios de selección.

En noviembre de 2011, se acogió en Buenos Aires la Agenda Prioritaria de Proyectos de Integración (API), cuyas modalidades se acordaron previamente en reunión de coordinadores nacionales de IIRSA, en agosto, en Río de Janeiro (Cosiplan-Unasur 2011).

Esta agenda articula corredores o espacios de integración y el Plan de Acción Estratégica (PAE) 2012-2022, con la identificación de 531 proyectos de integración, de los cuales 31 "proyectos estructurados" que consolidan redes de conectividad física con alcance regional para potenciar sinergias existentes y 88 proyectos individuales en la Agenda Prioritaria de Proyectos de Integración (API), que suman inversiones por US\$ 13.652,7 millones (Cosiplan-Unasur 2011).

Los proyectos corresponden a centros binacionales de atención en frontera y pasos de frontera, a interconexiones fronterizas, ejes viales, ferroviarios, corredores viales y ferroviarios, centros logísticos y puertos, navegabilidad de ríos, construcción de puentes, túneles, aeropuertos, transporte multimodal y gasoductos y líneas de transmisión.

Finalmente, al revisar mandatos recientes del Consejo, se puede verificar el cambio producido con el propósito de disponer de instrumentos que faciliten una mayor participación de las poblaciones de las regiones involucradas en los proyectos de IIRSA (Cosiplan-Unasur 2012a).

Es así como en el Plan de Trabajo 2013 enmarcado en acciones del PAE 2012-2022, se precisan acciones que incorporan la problemática de las regiones involucradas como las siguientes:

Elaborar y aplicar una metodología que permita evaluar la mejora de la calidad y esperanza de vida de las poblaciones como consecuencia de la implementación de los proyectos de infraestructura y recomendar acciones futuras.

Definir instancias de participación social y de contribución activa de las comunidades involucradas en las actividades del Cosiplan, a partir de los lineamientos definidos por la Unasur conforme su Tratado Constitutivo.

Realizar un intercambio de información y experiencias entre los países sobre sus respectivos mecanismos de participación ciudadana, a través de la Presidencia Pro Tempore.

134 Si Somos Americanos. Revista de Estudios Transfronterizos 
Coordinar con otras instancias de Unasur para definir los mecanismos de participación social, lo cual permitirá identificar las acciones correspondientes (Cosiplan-Unasur 2012b).

Este tipo de acciones para una mayor participación de las comunidades locales se complementa en el terreno del Planeamiento Territorial Indicativo, con mandatos para el 2013 como este: "Revisar y perfeccionar la metodología y el proceso de planeamiento territorial indicativo, teniendo como referencia los objetivos definidos en el marco del Cosiplan" (Cosiplan-Unasur 2012b).

Y en los programas territoriales de integración: "Definir una metodología para la creación de Programas Territoriales de Integración (PTI) que complementen la Agenda de Proyectos Prioritarios de Integración (API)" (Cosiplan-Unasur 2012b).

Se puede apreciar cómo se comienza a hablar un lenguaje más esperanzador de que las políticas de desarrollo de las regiones se vinculen a las nuevas acciones de infraestructura de un órgano técnico como IIRSA, enmarcándolo en un contexto más político como es Cosiplan en la estructura de consejos sectoriales de la Unión de Naciones Suramericanas (Unasur).

\section{CONCLUSIONES}

El actual proceso de globalización crea condiciones favorables para atender programas de desarrollo de regiones en una reconceptualización de lo territorial, en una modalidad específica de desarrollo referida al "desarrollo económico local". De esta manera se reelaboran conceptos como los de distritos industriales, nociones como las de medios innovadores, estrategias de especialización flexible y complejos productivos o clusters.

La superación de barreras fronterizas en la inmensidad del espacio suramericano es condición sine qua non para lograr la integración desde las regiones y recuperar el atraso en concretar los proyectos de integración transfronteriza, que al superar la consideración de las fronteras como barreras, permiten derramar los efectos benéficos en espacios ampliados que responden a sinergias de poblaciones locales.

En el desarrollo de políticas integracionistas basadas en las regiones, Suramérica deberá consolidar esfuerzos basados no solamente en el papel de las regiones, sino el de las ciudades y las ciudades-región, ante la tendencia mundial de urbanización. 
La infraestructura es tal vez el más político de los instrumentos de la integración, al hacer partícipes a los gobernantes y a la ciudadanía de propósitos de desarrollo comunes y una acción integrada de transporte, energía y comunicaciones como oferta de infraestructura, y permitirá el desarrollo tanto de sectores como de regiones.

El alto impacto para la integración física regional de los proyectos nacionales, binacionales o multinacionales de la Agenda Prioritaria de Proyectos de Integración (API) de Unasur-Consiplan, posibilitaría estructurar programas de desarrollo de las regiones suramericanas en espacialidades de alcances y participaciones diferentes.

En la planificación territorial se pueden estructurar como programas territoriales de integración, la incorporación de programas de sostenibilidad económica, ambiental y social, mejorando los impactos a nivel local de la infraestructura.

El respaldo político de la Unasur debe facilitar el desarrollo de los proyectos y mayores compromisos de participación ciudadana, así como la incorporación de cadenas productivas regionales de mayor valor agregado e intensidad tecnológica en los ejes de integración y desarrollo, que hagan realidad los planes de infraestructura de la IIRSA.

\section{REFERENCIAS}

1. Alburquerque, Francisco. 2001. "La política de desarrollo económico local." Pp. 21-45 en Desarrollo económico local y descentralización en América Latina: análisis comparativo, compilado por Aghon, Alburquerque y Cortés. Santiago: Proyecto Regional de Desarrollo Económico Local y Descentralización Cepal/GTZ.

2. Asamblea de las Regiones de Europa (ARE). 1996. Declaración sobre el regionalismo en Europa. Basilea: ARE.

3. Benko, George y Alain Lipietz (eds.). 1992. Les régions qui gagnent. París: PUF.

4. Berretoni, Daniel y Jorge Lucángeli. 2012. "Mercosur: las asimetrías y el Fondo para la Convergencia Estructural del Mercosur (FOCEM)." Integración \& Desarrollo 16 (34): $33-44$.

5. Boisier, Sergio. 1994. "Crisis y alternativas en los procesos de regionalización." Revista de la Cepal (52): 179-90. 
6. Braudel, Fernand. 2005. El Mediterráneo y el mundo mediterráneo en la época de Felipe II. México: Fondo de Cultura Económica, tomos I y II, sexta reimpresión.

7. Brunner, José Joaquín. 1998. Globalización cultural y posmodernidad. Santiago: Breviarios FCE.

8. Bustamante, Ana Marleny y Marina Sierra (coords.). 2004. Propuesta de definición y delimitación de la Zona de Integración Fronteriza: Área Norte de Santander (Colombia) Táchira (Venezuela). San Cristóbal: CEFI, Universidad de los Andes, Universidad Libre y Universidad Francisco de Paula Santander.

9. Comunidad Andina. 2006. Creación de la Red Andina de Ciudades. Quito: Declaración de Quito.

10. -----. 2004. Creación del Consejo Consultivo Andino de Autoridades Municipales. Lima: Comisión.

11. Communautés Européennes. 1998. La réforme des Fonds Structurels. Bruselas: Comité des Régions.

12. Comunidades Europeas. 2007. La política de cohesión 2007-2013. Comentarios y textos oficiales. Luxemburgo: Oficina de Publicaciones Comunidades Europeas.

13. Consejo Andino de Ministros de Relaciones Exteriores. 1999. Política comunitaria para la integración y desarrollo fronterizo. Lima: Decisión 459.

14. Consejo Mercado Común Mercosur. 2004. Foro consultivo de Municipios, Estados Federados, Provincias y Departamentos del Mercosur. Montevideo: Decisión № 41/04.

15. Cosiplan - Unasur. 2011. Agenda de Proyectos Prioritarios de Integración - API. Documento IIRSA. Buenos Aires: noviembre.

16. -----. 2012a. Informe de Avance. III Reunión Ordinaria del Cosiplan. Lima: 16 de noviembre.

17. -----. 2012b. Plan de Trabajo 2013. III Reunión del Consejo Suramericano de Infraestructura y Planeamiento Cosiplan/Unasur. Lima: 16 de noviembre.

18. Dollfus, Olivier. 1993. "Une lecture géographique du système monde." Cahier du GEMDEV 20: 43-53.

19. Fazio, Hugo. 2002. La globalización en su historia. Bogotá: Universidad Nacional de Colombia.

20. Giddens, Anthony. 1994. Les conséquences de la modernité. París: L’Harmattan. 
21. González, José. 1995. "La política regional de la Unión Europea. Integración fronteriza y política regional: marco general en la Unión Europea." Seminario La integración fronteriza y el papel de las regiones en la Unión Europea y en el Cono Sur: experiencias, opciones y estrategias. Montevideo: CEFIR: 3-16.

22. Guimaraes, Roberto. 2001. "Fundamentos territoriales y biorregionales de la planificación.” Cepal, Serie Medio Ambiente y Desarrollo (39): 1-78.

23. Held, David y Anthony McGrew. 2003. Globalización/Antiglobalización. Sobre la reconstrucción del orden mundial. Barcelona: Editorial Paidós.

24. Helleiner, Eric. 2000. "Reflexiones braudelianas sobre la globalización económica: el historiador como pionero." Análisis Político (39): 3-14.

25. Hirschman, Albert. 1958. The strategy of economic development. New Haven: Yale University Press.

26. IIRSA. 2011. IIRSA Diez años después: sus logros y desafíos. Buenos Aires: BID - INTAL.

27. Jessop, Bob. 2004. "La economía política de la escala y la construcción de las regiones transfronterizas." EURE 29 (89): 25-41.

28. Keating, Michael. 1996. Naciones contra el Estado - El nacionalismo de Cataluña, Québec y Escocia. Barcelona: Editorial Ariel.

29. Krugman, Paul. 1992. Geografía y comercio. Barcelona: Antonio Bosh editor.

30. Lahorgue, María Alice. 1997. Dinâmica espacial da produção e da população e as zonas de fronteira. Des restructurations industrielles et territoriales et nouveaux roles de l'Etat face aux défis de la mondialisation de l'économie. Grenoble: Université Pierre Mendés-France.

31. Loyer, Bárbara y José Luis Villanova. 1999. "États et souverainetés en Europe: l’exemple catalan." Hérodote, revue de géographie et de géopolitique 95: 21-34.

32. Mabileau, Albert. 1998. "La région à l'épreuve des relations intergouvernementales." Pp. 53-65 en Régions la croisée des chemins. Perspectives françaises et enjeux européens, editado por Delpoirier Elisabeth. París: Presses de Sciences Po.

33. Marks, Gary. 1993. "Structural Policy and Multilevel Governance in the EC." Pp. 391-410 en The State of the European Community, editado por A. Cafruny y G. Rosenthal, vol. 2. Boulder Colorado: Lynne Rienner. Londres: Logman. 
34. Mercosur. 2005. Fondo para la Convergencia Estructural del Mercosur (FOCEM). Montevideo: Decisión № 18.

35. Moncayo, Edgard. 2002. Nuevos enfoques teóricos, evolución de las políticas regionales e impacto territorial de la globalización. Serie Gestión Pública 25. Santiago: ILPES, Cepal.

36. Myrdal, Gunnar. 1959. Teoría económica y regiones subdesarrolladas. México: Fondo de Cultura Económica.

37. Ohmae, Kenichi. 1997. El fin del Estado-nación. El ascenso de las economías regionales. Santiago: Editorial Andrés Bello.

38. ----. 2005. El próximo escenario global. Desafíos y oportunidades en un mundo sin fronteras. Bogotá: Editorial Norma.

39. Perroux, François. 1955. "Notes sur la notion de pôle de croissance." Économie Appliquée 1-2.

40. Porter, Michael. 1991. La ventaja competitiva de las naciones. Buenos Aires: Javier Vergara Editor.

41. Prebisch, Raúl. 1951. "Crecimiento, desequilibrio y disparidades: interpretación del proceso de desarrollo.” Pp. 3-89 en Estudio económico de América Latina 1949, editado por Naciones Unidas. Estados Unidos: New York.

42. Rostow, W.W. 1963. Las etapas del crecimiento económico. México: Fondo de Cultura Económica.

43. Scott, Allen. 2001. Les régions et léconomie mondiale. La nouvelle géopolitique globale de la production et de la compétition économique. París: Editions l'Harmattan.

44. Siroen, Jean-Marc. 2004. La régionalisation de l'économie mondiale. París: Éditions La Découverte.

45. Steger, Manfred. 2009. Globalization. A very short introduction. Nueva York : Oxford University Press.

46. Veltz, Pierre. 1996. Mondialisation, villes et territoires: l'économie d’archipel. París: Presses Universitaires.

47. Vieira, Edgar. 2008. La formación de espacios regionales en la integración de América Latina. Bogotá: Convenio Andrés Bello y Universidad Javeriana. 
48. ----. 2012. La globalización en un mundo en transformación. Bogotá: Editorial CESA.

49. Viner, Jacob. 1950. The Custom Union Issue. Nueva York: The Carnegie Endowment for International Peace.

140 Si Somos Americanos. Revista de Estudios Transfronterizos 